\title{
Holistic but with reduced right-hemisphere involvement: The case of dyslexia in Chinese character recognition
}

\author{
Ricky Van Yip Tso ${ }^{1,2}$ - Ronald Tsz Chung Chan ${ }^{1}$ - Janet Hui-wen Hsiao ${ }^{3}$
}

Published online: 6 March 2020

(C) The Psychonomic Society, Inc. 2020

\begin{abstract}
Recent research on visual object recognition has suggested that the right hemisphere can engage either holistic or part-based processing depending on whether the recognition relies on configural (exact distances among features) or featural information, respectively. Consistent with this finding, expert Chinese reading has been marked by a left-side bias (an indication of righthemisphere lateralization) with decreased holistic processing (as assessed using the composite paradigm) due to its reliance on featural information. Here we examine two common perceptual expertise phenomena in object recognition - holistic processing and left-side bias - of Chinese characters in adolescents with developmental dyslexia and matched controls. We found that those with dyslexia showed stronger holistic processing, a weaker left-side bias, and worse performance in Chinese character dictation than controls. This was in contrast to Limited writers (proficient readers with limited writing experience) reported in Tso, Au, and Hsiao (Psychological Science, 25, 1757-1767, 2014), who showed stronger holistic processing and worse dictation performance, but the same level of left-side bias as controls. This result demonstrated two different perceptual mechanisms underlying holistic processing: Limited writers' holistic processing may be due to difficulties in de-emphasizing configural information unimportant to Chinese characters, whereas readers with dyslexia may have deficits selectively attending to character components to form appropriate part-based representations in the right hemisphere.
\end{abstract}

Keywords Perceptual expertise $\cdot$ Chinese-character recognition $\cdot$ Holistic processing $\cdot$ Dyslexia $\cdot$ Left-side bias

\section{Introduction}

Holistic processing (HP) is a concept originally derived from Gestalt psychology that postulates that the perception of an object as a whole is qualitatively different from the sum of its parts. HP has been commonly observed in face perception one example is the composite face illusion, in which two identical top half-faces are judged to be different when they are paired with different bottom halves. This illusion reflects

Ricky Van Yip Tso

rvytso@eduhk.hk

Janet Hui-wen Hsiao

jhsiao@hku.hk

1 Department of Psychology, The Education University of Hong Kong, Ting Kok, Hong Kong

2 Psychological Assessment and Clinical Research Unit, The Education University of Hong Kong, Ting Kok, Hong Kong

3 Department of Psychology, The University of Hong Kong, Pok Fu Lam, Hong Kong obligatorily attention to all facial features as a whole (Richler, Wong, \& Gauthier, 2011). Beyond face perception, some have posited that expert subordinate-level object recognition requires HP (Bukach, Gauthier, \& Tarr, 2006).

Chinese characters share many visual properties with faces. They have a homogenous, square configuration - with each character a grapheme mapping onto a morpheme. Strokes are the basic units of the orthography, which combine to form more than 200 basic stroke patterns (Hsiao \& Shillcock, 2006), which in turn form the characters. A person can recognize different faces regardless of their facial expressions, similar to a literate needing to recognize over 3,000 characters regardless of fonts. Hence, theoretically, Chinese characters should induce similar perceptual expertise effects as faces do (Hsiao \& Cottrell, 2009).

However, in contrast to the literature suggesting HP as a perceptual expertise marker, Hsiao and Cottrell (2009) found that expert Chinese readers had a reduced HP effect compared with novices. Tso, Au, and Hsiao (2014) further showed that this reduced HP effect in expert readers may be explained by writing performances: Compared with novices, proficient 
readers with limited writing performances (Limited-writers) showed increased HP, while expert readers with typical writing abilities (Writers) showed reduced HP. These findings hint at a modulating role of writing abilities in HP. Tso et al. (2014) also found that, while Limited-writers had comparable character naming accuracy to Writers, they had significantly longer response times, suggesting less efficient character processing. Thus, expert Chinese character recognition involves the ability to selectively attend to and efficiently identify character components, as demonstrated in the reduced HP effect.

Left-side bias (LSB) is another perceptual phenomenon commonly reported in face perception: A chimeric face created by fusing two left half-faces together is often judged more similar to the original face than one created by fusing two right half-faces (from the viewer's perspective; Brady, Campbell, \& Flaherty, 2005; Fig. 1). The LSB effect has also been demonstrated in expert Chinese character recognition and is suggested to be associated with right-hemisphere $(\mathrm{RH})$ involvement in face and Chinese character recognition (Brady et al., 2005; Hsiao, Shillcock, \& Lee, 2007).

Though LSB, or RH lateralization, has been thought to correlate with increase in HP in visual object recognition (Gauthier \& Tarr, 2002), expert Chinese character recognition has been reported to involve increased LSB and decreased HP, presenting a counter example (Hsiao \& Cottrell, 2009). Tso et al. (2014) added that LSB is a consistent expertise marker for Chinese characters unaffected by readers' writing experiences, since Writers and Limited-writers showed a similar level of LSB. Through both computational modeling and human subject experiments with face stimuli, Hsiao and Galmar (2016) showed that the relationship between RH lateralization and HP in visual object recognition depends on whether the recognition task relies on featural information or configural information (i.e., exact distances, or second-order relations among features; see Maurer, Grand, \& Mondloch, 2002): a positive correlation was observed when the task relies purely on configural information, whereas a negative correlation was found when the task relies purely on featural information. In Chinese character recognition, featural information (e.g., individual strokes) is more critical to character identification than configural information (e.g., exact distances between strokes). Accordingly, HP and LSB in Chinese character-recognition expertise would not go together, consistent with the finding of decreased HP and increased LSB reported in Hsiao and Cottrell (2009). Chung, Leung, Wong, and Hsiao (2018a) further showed that when Chinese characters were presented to the left visual field/RH, Chinese novice readers showed HP effects whereas expert readers did not. This suggests that the RH may flexibly switch between holistic and part-based representations, consistent with a recent fMRI study (Harris \& Aguirre, 2010). Chung et al. (2018) argued that the advantage of the RH in low spatial frequency processing is relative to the task-relevant frequency range (Ivry \& Robertson, 1998), which depends on whether attention is allocated to the whole stimulus or to the parts. Thus, the $\mathrm{RH}$ can be involved in forming a low spatial frequency biased representation for either the whole or a part of the stimulus based on the task demand.

The understanding of the relationship between HP and RH lateralization gives us important insights into the mechanism underlying deficits in visual object recognition. For example, patients with prosopagnosia (clinical difficulties in face recognition) were reported to have reduced HP (Avidan, Tanzer, and Behrmann, 2011) and LSB in face perception (Malaspina, Albonic, \& Daini, 2016). These results suggest that their deficits may be related to difficulties in forming appropriate configural representations in the RH. In contrast, HP and LSB effects in Chinese readers with reading difficulty (dyslexia) remain unclear. Difficulties in Chinese character recognition may be related to failure to de-emphasize configural information unimportant for character identification; this may lead to LSB with increased HP as compared with typically developing readers, such as the Limited-writers reported in Tso et al. (2014). Alternatively, if Chinese dyslexics show increased HP and reduced LSB as compared with controls, it would suggest that their deficits may be related to inability to selectively attend to character parts to form appropriate part representations in the $\mathrm{RH}$ rather than inability to deemphasize configural information.
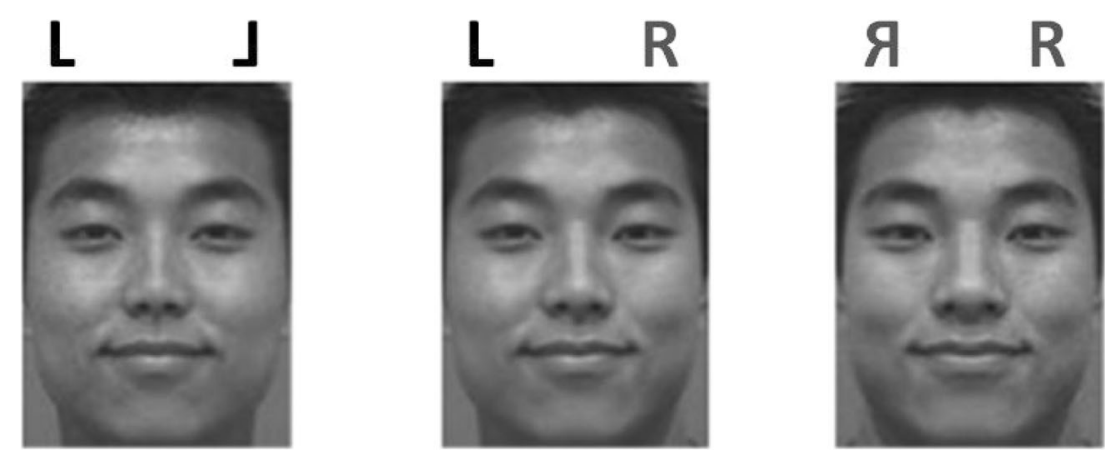

Fig. 1 Examples of chimeric face stimuli (adopted from Hsiao \& Cottrell, 2009). The left chimeric face was formed by combining two left halves of an original face (middle), while the right chimeric face was formed by the two right halves 
While dyslexia in alphabetic languages is generally associated with core deficits in phonological skills and corresponding neurological deficits, dyslexia in Chinese is more associated with abnormalities in orthographic or visuospatial processing (Siok et al., 2004). In particular, character writing and copying abilities play an important role in Chinese literacy development (Tan et al., 2005), and children with Chinese reading difficulties often have a marked discrepancy between reading and writing abilities (Chung \& Ho, 2010). As writing abilities seem to be particularly relevant to the reduced HP effect in expert Chinese readers (Tso et al., 2014), people with dyslexia may fail to employ analytic (reduced holistic) processing. In addition, Siok et al. (2004) showed reduced activities in the RH during Chinese character processing in dyslexics when compared with controls. Together these findings suggest that Chinese readers with dyslexia may show increased HP and reduced LSB in character perception as compared with controls, which would suggest their inability to selectively attend to character parts to form appropriate part representations in the RH. Here we tested this hypothesis through recruiting adolescents in Hong Kong who were native Chinese speakers and readers with and without dyslexia and examining their difference in HP and LSB in Chinese character perception.

\section{Methods}

\section{Participants}

Twenty typically developing students and 20 students diagnosed with dyslexia were recruited from a secondary school in Hong Kong, with four females and 16 males in each group. This sample size was determined by taking into consideration the sample size in the Tso et al. (2014) study, in which a large effect could be detected with a sample size of 40 participants. Dyslexia in Hong Kong is typically diagnosed by a clinical or educational psychologist using standardized test batteries as having normal IQs and performing at least one standard deviation below their same-age peers in the literacy domain and at least one literacy-relative cognitive subtest using the Hong Kong Test of Specific Learning Difficulties in Reading and Writing for Primary School Students Third Edition [HKT-P(III)] (Ho et al., 2016). Participants with dyslexia had been identified and diagnosed by a qualified psychologist prior to joining this study, and all reported no other co-morbid learning disabilities. All of them reported normal or corrected-to-normal vision. The two groups were matched in age $(\mathrm{M}=14.6$ years, S.E. $=$ .26), $\mathrm{F}(1,39)=1, \mathrm{p}=.753$.

\section{Materials and procedures}

Chinese literacy Dictation and Chinese word reading performance in both timed and untimed contexts were measured as a reference for the participants' literacy performances. The stimuli were adopted from HKT-P(III). Previous pilot studies have shown that the HKT-P(III) could even differentiate college students with dyslexia from those without. As our purpose was not to yield diagnostic results, the use of the stimuli from HKT-P(III) was for research purposes only to provide a comparison of literacy performances between students with dyslexia and those without.

The subtests administered were: (1) the Untimed Chinese word reading task, which assessed word reading accuracy; (2) the Chinese one-minute word reading task, which assessed word reading fluency; and (3) the Chinese dictation task, which assessed word-writing ability.

Orthographic awareness The lexical decision task was adopted from Ho et al. (2016) to assess students' performance in discriminating orthographically legal characters from illegal ones. Their response time to finish the task was measured.

Rapid automatized naming Because general symbolprocessing speed is related to reading ability in children (Wolf \& Bowers, 1999), we used the Rapid Digit Naming task from HKT-P(III) to measure rapid automatized naming (RAN) of symbol-processing speed. This test contains eight rows of five digits $(2,4,6,7,9)$ arranged in a random sequence in each row. Participants were instructed to read the digits aloud as quickly and accurately as they could. Each participant attempted the task twice and the score was calculated by the average time to complete.

Nonverbal intelligence To control for the effect of IQ on reading, nonverbal intelligence was assessed using the nine-item subset of Raven's standard progressing matrices (Bilker et al., 2012).

Holistic processing Following Hsiao and Cottrell (2009) and Tso et al. (2014), the complete composite paradigm was used to assess HP effects. 160 pairs of medium- to high-frequency Chinese characters in Ming font were used - half of the pairs in a top-bottom configuration and the other half in a left-right configuration (Tso et al., 2014; ${ }^{1}$ Fig. 2). Forty pairs were presented in each of the four conditions in Fig. 3a. In the congruent trials, the attended halves and the irrelevant halves led to the same response. In the incongruent trials, the attended halves and the irrelevant halves led to different responses.

\footnotetext{
${ }^{1}$ Analysis of a Chinese character database suggests that about $80 \%$ of characters are of either top-bottom or left-right configurations (Hsiao \& Shillcock, 2006). Hence, left-right configurations were added in this study to have a better representation of the Chinese lexicon. See Liu, Chuk, Yeh, and Hsiao (2016).
} 

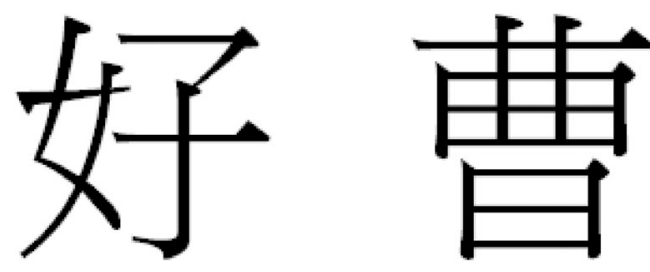

Fig. 2 Examples of Chinese characters with a left-right configuration (left) and a top-bottom configuration (right)

The same procedure as that in Tso et al. (2014) was used (Fig. 3b). Participants' performance in each condition was measured by discrimination sensitivity $\mathrm{A}^{\prime}$ as:

$A^{\prime}=0.5+\left[\operatorname{sign}(H-F) \frac{(H-F)^{2}+|H-F|}{4 \max (H, F)-4 H F}\right]$

where $\mathrm{H}$ and $\mathrm{F}$ are the hit and false-alarm rate, respective. $\mathrm{A}^{\prime}$ was used due to its bias-free nonparametric property (Hsiao \& Cottrell, 2009). HP was measured by A' or response time difference between the congruent and incongruent trials - the larger the discrepancy, the larger the HP effect. In addition, a misaligned condition was included such that if the HP effect was due to interference from the irrelevant halves, misalignment would reduce this effect (Fig. 3b).

Left-side bias The procedure from Tso et al. (2014) was adopted (Fig. 4). Eighty high-frequency mirror-symmetric Chinese characters were used. Each character was presented once in Ming font. Half of the trials displayed the original characters, whereas the other half displayed characters in mirror image; this was counter-balanced across participants. For each stimulus, two left halves constructed the left chimeric character while two right halves formed the right chimeric character. LSB was measured as the percentage of trials in which participants selected the left chimeric character as more similar to the original character.

\section{Results}

\section{Literacy abilities and non-verbal intelligence}

Separate independent sample $t$-tests were carried out to examine the effect of group (Dyslexics vs. Control) on each literacy test (Tables 1 and 2). Participants with dyslexia and matched controls had similar performances in Chinese word reading and 1-min word reading. However, the controls had marginally better performance in Chinese word dictation, suggesting that the students with dyslexia had persistent difficulties in writing Chinese characters even in high-school grades. The participants with dyslexia were also slower in RAN than the controls, suggesting that they had persistent deficits in automatized naming of orthographic symbols. The controls also had significantly faster response times in lexical decision than the dyslexics, suggesting the dyslexics were slower at discriminating orthographically legal characters from orthographically illegal ones. ${ }^{2}$

Holistic processing We conducted a 2 (congruency: congruent vs. incongruent) $\times 2$ (group: dyslexics vs. control) repeatedmeasures ANOVA on the data in the aligned condition to examine HP effects. In A', there was a main effect of congruency, $F(1,38)=27.35, p=.000006, \eta p^{2}=.419$, but no interaction between congruency and group, $\mathrm{F}(1,38)=1.354, \mathrm{p}=$ .252 , or main effect of group, $F(1,38)=1.342, p=.254$. In response time, we found a significant interaction between congruency and group, $\mathrm{F}(1,38)=5.854, \mathrm{p}=.02, \eta \mathrm{p}^{2}=.133$, and a main effect of group, $\mathrm{F}(1,38)=5.306, \mathrm{p}=.027, \eta \mathrm{p}^{2}=.123$, but no main effect of congruency, $\mathrm{F}(1,38)=2.254, \mathrm{p}=.150$. Post hoc repeated-measures ANOVA showed that students with dyslexia responded more slowly in incongruent than in congruent trials, $\mathrm{F}(19)=34.3, \mathrm{p}<.000012, \eta^{2}=.644$, whereas response times in congruent and incongruent trials were similar in typically developing students, $\mathrm{F}(19)=0.254, \mathrm{p}=$ .620 (Fig. 5). To examine whether the congruency effect observed in the students with dyslexia was indeed due to interference from the irrelevant halves, following Hsiao and Cottrell (2009), we conducted a 2 (congruent vs. incongruent) $\times 2$ (aligned vs. misaligned) repeated-measures ANOVA in the two participant groups separately. The results showed that misalignment significantly reduced the congruency effect demonstrated in response time in the students with dyslexia: a significant interaction was found between congruency and misalignment, $F(1,19)=5.662, p=.029, \eta p^{2}=.239$. There was no misalignment effect in typically developing students. These results suggest that participants with dyslexia perceived Chinese characters more holistically than the controls. ${ }^{3}$

\footnotetext{
${ }^{2}$ At the moment, there is no standardized test or normed test to assess literacy performance for adolescents and adults in Hong Kong aged 15.5 years and above. The literacy test stimuli in this study were obtained from HKT-P(III), and had been tested previously in a pilot study to be able to differentiate between college students with dyslexia and those without. While the reading performance for both groups of participants were high, the scores for dictation, lexical decisions, and RAN were able to effectively differentiate students with and without dyslexia. These results echoed those of other studies on dyslexia in the Chinese population that have suggested persistent writing difficulties associated with RAN and orthographic awareness as they progress to adolescence and adulthood (Chan et al., 2006; Chung et al., 2011; Chung, Lo, \& Mcbride, 2018).

${ }^{3}$ Note that in contrast to the partial design in which holistic processing is measured by alignment effect (i.e., the performance discrepancy between aligned and misaligned conditions; e.g., Hole, 1994; Robbins \& McKone, 2007), the complete composite design measures holistic processing by congruency effect indicated by the performance difference between the congruent and incongruent trials without a misalignment condition (see Gauthier \& Bukach, 2007; Hsiao \& Cottrell, 2009; Tso et al., 2014). Hsiao and Cottrell (2009) showed that the congruency effect observed in Chinese character processing disappeared when character halves were misaligned, consistent with the current results as well as the literature on face and object recognition (see Richler, Tanaka, Brown, \& Gauthier, 2008).
} 


\section{Left-side bias}

The results showed that the typical readers had a stronger LSB than the students with dyslexia, $F(1,38)=6.439, p=.015, \eta p^{2}$ $=.145$ (Fig. 6). Thus, although those with dyslexia were more holistic than the typical readers in Chinese character recognition, they revealed a weaker LSB. ${ }^{4}$

\section{Discussion}

This study investigated how high-school students with dyslexia and those without differed in HP (using the composite paradigm) and LSB in Chinese character perception in order to understand the mechanism underlying the deficits in Chinese dyslexia. Recent research has suggested that the RH has the flexibility to switch between holistic and part-based representations in visual object recognition, depending on whether the recognition relies on configural or featural information, respectively (Chung et al., 2018a, b; Hsiao \& Galmar, 2016). Consistent with this finding, expertise in Chinese character recognition, where featural information plays a more important role, is associated with decreased HP and increased LSB/RH lateralization (Hsiao \& Cottrell, 2009; Tso et al., 2014). Accordingly, if reading difficulty in students with dyslexia was associated with inability to de-emphasize configural information unimportant for Chinese character identification, they would show increased HP together with LSB. In contrast, if they showed increased HP together with decreased LSB, it would suggest that their deficit was in the inability to attend to

\footnotetext{
${ }^{4}$ To examine which literacy-related variables could better explain the difference in HP and LSB between the dyslexics and controls, we used ANCOVA to examine the congruency effect in RT. With age, Raven's score, and all the literacy-related variables (RAN, Chinese word-reading, 1-min word reading, dictation, and lexical decision performances) entered as covariates, a significant difference was still found for HP, $F(1,38)=12.848, p=.001$. and LSB, $\mathrm{F}(1,38)=4.805, \mathrm{p}=.036$. These effects suggested that the observed differences between dyslexics and controls in HP and LSB were better explained by their diagnostic condition, though they were in concurrence with the differences in dictation, lexical decision, and RAN.

In a separate analysis, we found that HP and LSB were not significantly correlated in the participants, $r=-.092$, n.s. (Table S1 in Supplementary Information). While computational studies have shown that HP and LSB are negatively correlated in recognition tasks that purely depend on featural information (e.g., Hsiao \& Galmar, 2016), real-life visual stimuli naturally embed both featural and configural differences. Although the recognition can depend more on either featural or configural information, typically both types of information are used. Thus, a strong correlation between HP and LSB using reallife stimuli is not always observed. Indeed, in Hsiao and Galmar's (2016) computational study, when using real face stimuli, LSB and HP did not correlate with each other, consistent their human data. In the current study, while HP did not correlate with the Chinese literacy measures, LSB was associated with lexical decision performance. This result suggests that some form of RH processing (which may be related configural information) is associated with the knowledge of legality of Chinese orthography, but not the ability to selectively attend to a component as measured in HP. This result also again suggests that HP and LSB are separate processes that do not always go together; it depends on the task demands (Hsiao \& Galmar, 2016).
}

character parts to form appropriate part-based representations in the RH.

Our results showed that participants with dyslexia demonstrated stronger HP and weaker LSB in Chinese character perception than typically developing controls. Thus, they may have deficits in selectively attending to character parts to form appropriate part-based representations in the RH. This finding was consistent with previous studies reporting weaker $\mathrm{RH}$ involvement in Chinese character recognition in Chinese dyslexia (Siok et al., 2004). These part-based representations may be important for readers to learn about first-order relations (relative positions) among character parts. In contrast to second-order relations (exact distances) among parts (see Maurer et al., 2002), the knowledge about first-order relations plays an essential role in Chinese character recognition. As Chinese character components are position-specific and often convey specific semantic or phonological information (Hsiao \& Shillcock, 2006), the awareness of first-order relations among components in a character, or orthographic awareness, allows readers to efficiently identify the meaning and pronunciation of the character. Consistent with this speculation, here students with dyslexia had a lower performance in lexical decision when compared with that in controls. Indeed, in previous studies, Chinese readers with dyslexia have often been found to have impaired orthographic awareness as compared with controls (Chung \& Ho, 2010).

Note that while both students with dyslexia and Limitedwriters in Tso et al.'s (2014) study demonstrated increased HP and low character dictation performance, they differed in LSB: Limited-writers showed LSB whereas students with dyslexia did not. This phenomenon suggests different mechanisms underlying their HP. Without adequate writing experience, Limited-writers may have less ability to de-emphasize configural information among features that are unimportant for Chinese characters, leading to increased HP together with LSB. In contrast, since writing practice has been regularly implemented in the curriculum for Chinese literacy in Hong Kong, students with dyslexia may have learned to deemphasize unimportant configural information but suffered from deficits in visually separating and attending to character components, leading to increased HP with decreased LSB. The current examination helped us to tease apart these two different mechanisms underlying HP. Consistent with this speculation, here we did not observe a significant correlation between HP and dictation performance among the participants (Supplementary Material, Table S1), in contrast to Tso et al.'s (2014) study that suggested a close relationship between HP and writing ability. This phenomenon again suggested different mechanisms underlying HP effects in Chinese character processing. Alternatively, it is possible that both Limitedwriters and students with dyslexia had deficits to deemphasize unimportant configural information, leading to increased HP, and the reduced LSB in students with dyslexia 


\section{Congruent trials}

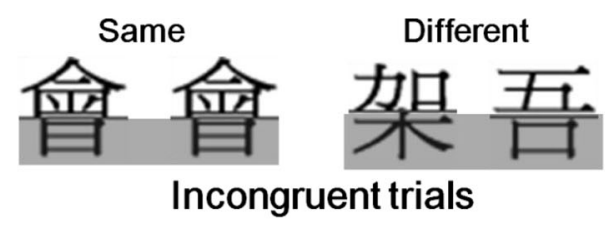

(a)
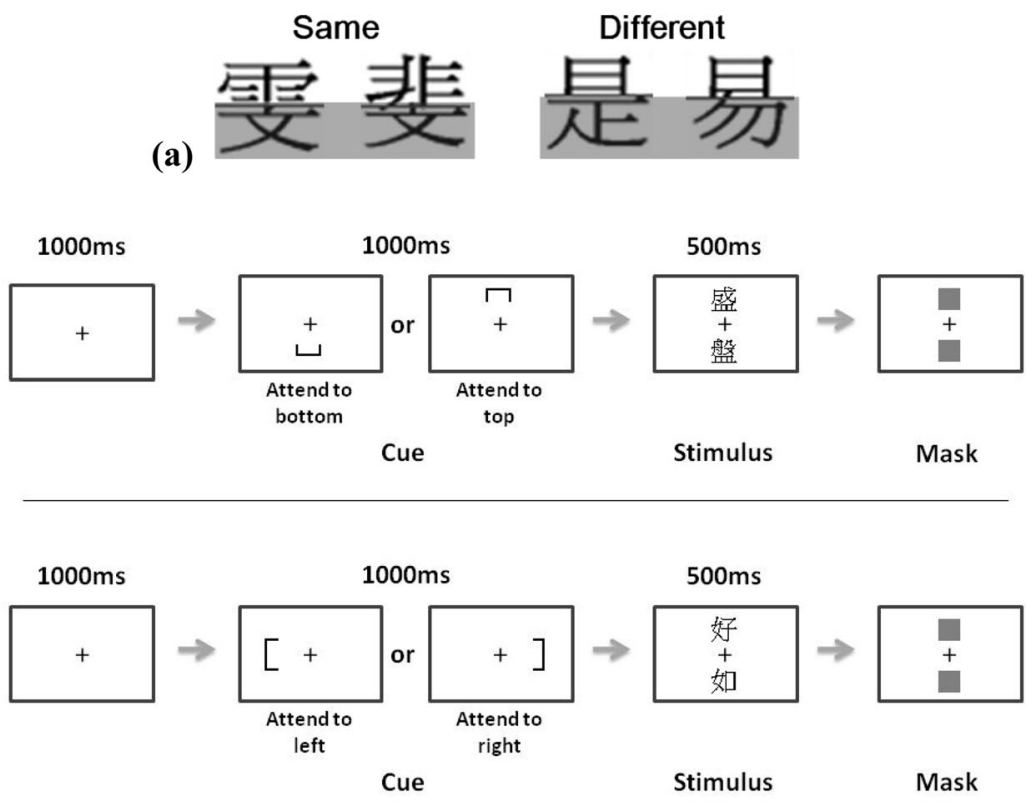

(b)

(c)

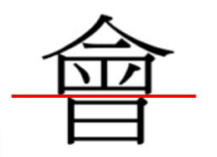

Fig. 3 Illustration of stimulus pairs in the complete composite paradigm and trial sequences. In (a), it shows the four conditions used in the paradigm; the attended components are shaded in grey. In (b), a 1,000ms central fixation cross preceded each trial, followed by a cue either

may be related to some other factors affecting the LSB effect, such as deficits in processing first-order relations among components. These possibilities require further investigations.

Our results suggest that interventions that involve enhancing the ability to visually separate and selectively attend to character components may help Chinese readers with dyslexia to develop appropriate representations for character parts and the first-order relations among them. This may in turn enhance orthographic awareness and reading performance. Interventions designed for young students with dyslexia often involve identifying and recognizing individual semantic and phonetic components within Chinese characters, which has been reported to significantly improve their performances in character recognition and writing (Tse, 2011). The weaker LSB and increased HP effects in the students with dyslexia could also be related to the possibility of reduced reading experience due to their reading difficulties. Indeed, recent research has shown that literacy experience modulates neural responses to written words (Dehaene, Cohen, Morais, \&

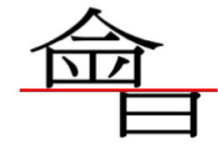

below or above the cross, or to the left or right of the cross, to indicate which halves (top or bottom/left or right) of the characters participants should attend to in the subsequent display. (c) A character in aligned (left) and misaligned conditions (right)

Kolinsky, 2015). Thus, adequate literature exposure may also play an important role in the interventions.

Franceschini, Bertoni, Gianesini, Gori, and Facoetti (2017) recently reported reduced global processing in people with dyslexia in Italian using hierarchical letter pattern stimuli (Navon, 1977). This result was consistent with our finding of a reduced LSB effect in Chinese dyslexia. Note, however, that Franceschini et al. (2017) based their study on Italian readers while the current study was on Chinese reading processes. In addition, the task using Navon stimuli measures participants' ability to attend to either the global form or the local components of the stimuli when all local components have the same identity, whereas the composite paradigm in the current study measures participants' ability to selectively attend to a stimulus part under the interference from surrounding parts of a different identity. It remains unclear whether English dyslexics would show increased HP effects with English word stimuli or whether Chinese dyslexics would show reduced global processing using Navon stimuli. 
a
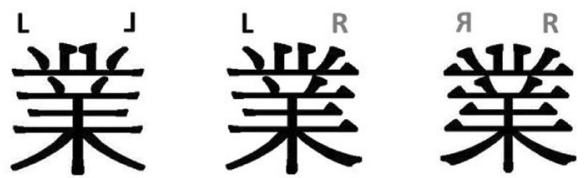

b

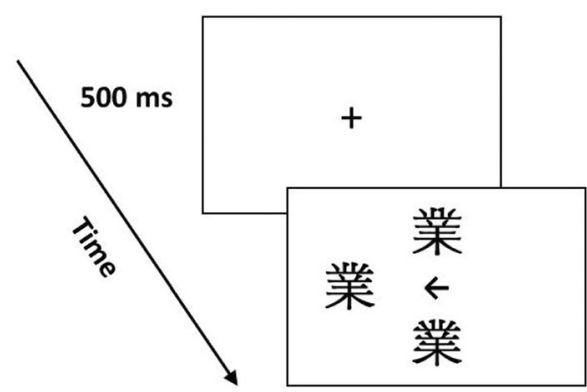

Fig. 4 (a) Examples of the stimuli, and (b) the test sequence in the LSB experiment (note that the chimeric characters are still legal Chinese characters). Each character spanned a visual angle of about $6.7^{\circ}$ with a $55-\mathrm{cm}$ viewing distance. After a 500-ms central fixation cross in each trial, the original character was displayed either on the left or right side of the screen randomly, at about $7.2^{\circ}$ of visual angle away from the center. Simultaneously, the left and right chimeric characters were presented with one above and one below an arrow at the screen center. The arrow pointed to the original character. Each chimeric character was about $3^{\circ}$ of visual angle away from the center. All stimuli were displayed until the participant responded, by pressing corresponding buttons on a response box, to judge which of the two chimeric characters looked more similar to the original one

Franceschini et al.'s (2017) finding suggested that dyslexics in alphabetic languages may have problems integrating letters to form a decodable word, whereas our results showed that dyslexia in Chinese may have impaired part processing in the RH. Together these results suggest that dyslexia may be associated with impaired RH processing in flexibly switching between holistic and part-based representations. This speculation is consistent with recent research suggesting that children with dyslexia may have deficits in coordination between analytic
Table 2 Results of independent-sample $t$-tests (dyslexics vs. Control) for Chinese word-reading, Chinese 1-min word reading, Chinese dictation, and non-verbal IQ (nine-item Raven's) in high-school students with and without dyslexia

\begin{tabular}{llll}
\hline & $t$ & $p$ & $d$ \\
\hline Chinese word reading & .735 & .476 & .24 \\
One-minute word reading & .941 & .353 & .31 \\
Chinese word dictation & 1.960 & .057 & .61 \\
Lexical decision & 2.229 & .031 & .73 \\
RAN & 2.722 & .01 & .88 \\
9-item Raven's score & .105 & .917 & .03 \\
\hline
\end{tabular}

and holistic processing needed for fluent reading (e.g., Lachmann, 2018; Schmitt, Lachmann, \& Leeuwen, 2019).

Consistent with the above discussions on the association between dyslexia and impairment in RH processing, children with dyslexia in both alphabetic languages (e.g., Bosse, Tainturier, \& Valdois, 2007; Facoetti, et al., 2001; Gabay, Gabay, Henik, Schiff, \& Behrmann, 2015; Gabay, Gabay, Schiff, Ashkenazi, \& Henik, 2013; Stein \& Walsh, 1997) and the Chinese language (e.g., Liu, Pan, \& Xu, 2018) were shown to have deficits in visual spatial attention. For example, Bosse et al. (2007) found that many British and French children with dyslexia exhibited deficits in visual attention span, i.e., the number of visual elements that can be processed simultaneously, in addition to phonological disorders. This impairment may influence recognition in other visual domains. Indeed, recent research has reported that individuals with dyslexia in an alphabetic script also showed impaired face processing, suggesting that cognitive processes in different domains may be interdependent (e.g., Behrmann \& Plaut, 2012, 2013; Gabay, Dundas, Plaut, \& Behrmann, 2017; Sigurdardottir, Arnardottir, Thuridur, Halldorsdottir, \& Valgeirsdottir, 2019). Future work will examine whether those with Chinese dyslexia also have deficits in face processing.

Table 1 Summary of the scores of Chinese word-reading, Chinese 1-min word reading, Chinese dictation, and non-verbal IQ (nine-item Raven's) in high-school students with and without dyslexia

\begin{tabular}{|c|c|c|}
\hline & Control Mean (SE) & Dyslexics Mean (SE) \\
\hline Chinese word-reading (\# correct responses out of 150) & $\begin{array}{l}102 \text { (1.82) } \\
\text { (Min: 85; Max: 118) }\end{array}$ & $\begin{array}{l}99.8 \text { (3.41) } \\
\text { (Min: 77; Max: 114) }\end{array}$ \\
\hline Chinese one-minute word reading (\# correct responses) & $\begin{array}{l}93.43 \text { (4.62) } \\
\text { (Min: 44; Max: 145) }\end{array}$ & $\begin{array}{l}85.91 \text { (3.95) } \\
\text { (Min: 56; Max: 127) }\end{array}$ \\
\hline Chinese dectation (\# correct responses out of 90) & $\begin{array}{l}59.73 \text { (13.14) } \\
\text { (Min: 36; Max: 79) }\end{array}$ & $\begin{array}{l}47.52 \text { (17.35) } \\
\text { (Min: 20; Max: 74) }\end{array}$ \\
\hline Lexical decision (responses time in seconds) & $\begin{array}{l}42.6(11.04) \\
\text { (Min: 17.01; Max: 63.1) }\end{array}$ & $\begin{array}{l}49.77 \text { (9.24) } \\
\text { (Min: 29; Max: 73.46) }\end{array}$ \\
\hline Rapid Automatized naming (response time in seconds) & $\begin{array}{l}13.0(.743) \\
\text { (Min: } 7.71 ; \text { Max: 18.72) }\end{array}$ & $\begin{array}{l}15.9(.743) \\
\text { (Min: } 7.72 \text {; Max: 21.64) }\end{array}$ \\
\hline Nan-verbal IQ (9-item Raven'; (performance score) & $\begin{array}{l}3.91 \text { (1.74) } \\
\text { (Min: } 1 ; \text { Max: } 8)\end{array}$ & $\begin{array}{l}3.96(1.34) \\
\text { (Min: } 1 \text {; Max: } 6)\end{array}$ \\
\hline
\end{tabular}




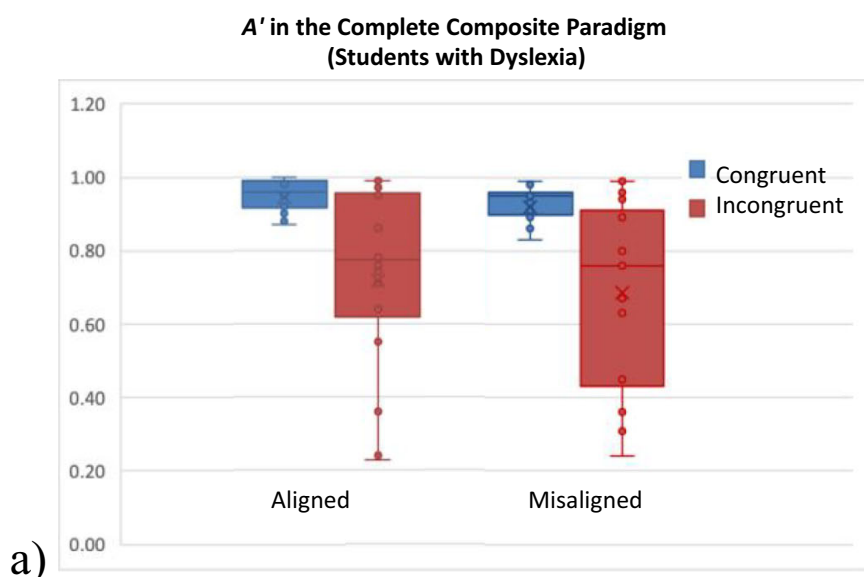

Response Time (ms) in the Complete Composite Paradigm (Students with Dyslexia)



c)

Fig. $5 \mathrm{~A}^{\prime}$ and response time (RT) in the complete composite paradigm are shown in (a, b, c, and $\mathbf{d})$. The holistic processing (congruency) effect in character perception was significant for dyslexics (c), but not the typically developing readers (d) as shown in the RT. A' did not reveal significant group differences as shown in (a) and (b). Misalignments significantly

\section{Probability of left-chimeric character chosen to resemble the original character}



Fig. 6 Left-side bias effect in Chinese character perception was larger in typically developing readers, but not in dyslexics. The intercepting line in the box denotes the group median, $x$ represents the mean, while the box denotes data points from the first to third quartiles. The range is represented by the whiskers

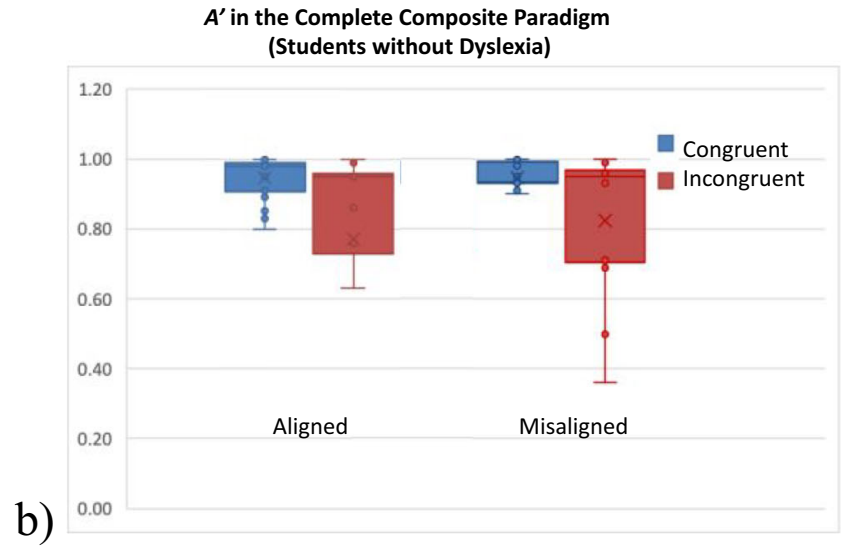

Response Time (ms) in the Complete Composite Paradigm (Students without Dyslexia)

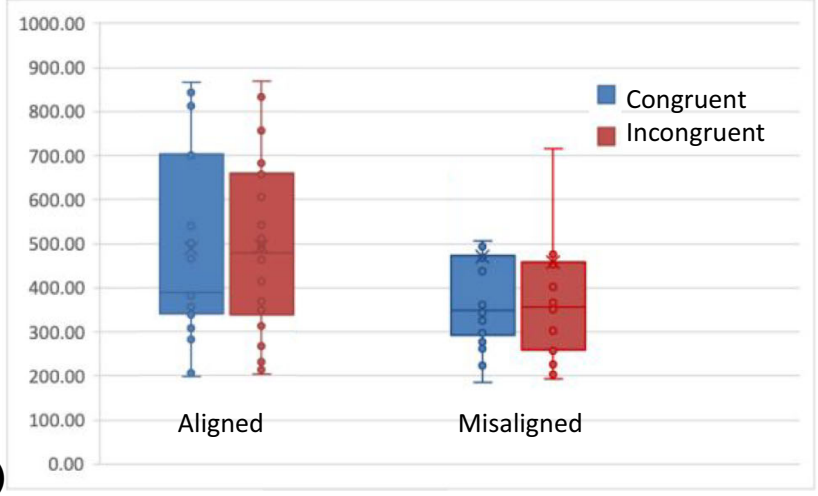

reduced the effect in the dyslexics as shown in RT. The intercepting line in the box denotes the group median, $x$ represents the mean, while the box denotes data points from the first to third quartiles. The range is represented by the whiskers

The inclusion of non-linguistic stimuli such as faces will also help us understand whether the observed LSB and HP effects are related to impaired linguistic processing.

In contrast to Chinese readers with dyslexia, people with prosopagnosia were reported to have reduced LSB (Malaspina et al., 2016) and HP (Avidan et al., 2011) in face perception. These findings suggest that the deficits may be related to difficulties in forming appropriate configural representations in the RH. Note however that recent research has suggested both configural and featural information are important for face recognition (Sandford \& Burton, 2014). The optimal strategy may involve the flexibility to switch between the use of configural and featural information (Chan, Chan, Lee, \& Hsiao, 2018; Chuk, Crookes, Hayward, Chan, \& Hsiao, 2017). Future work may examine HP and LSB in prosopagnosia patients to better understand whether deficits in forming either configural or part-based representations in the $\mathrm{RH}$ can lead to face recognition difficulties. 
To conclude, here we have shown that Chinese readers with dyslexia had increased HP, decreased LSB, and worse dictation performances on Chinese characters as compared with typically developing controls. This result was in contrast to Limited writers reported in Tso et al. (2014), who showed increased HP and worse dictation performance with a significant LSB, demonstrating two different perceptual mechanisms underlying HP. Recent studies have suggested that the RH can switch between holistic and part-based representations depending on whether the recognition relies on configural or featural information respectively (Chung et al., 2018a, b; Harris \& Aguirre, 2010). As Chinese character recognition relies more on featural than configural information, Limited writers' HP with LSB suggests their difficulties in deemphasizing configural information unimportant to Chinese character recognition due to inadequate writing experience. In contrast, the stronger HP with reduced LSB in readers with dyslexia suggests their deficits in selectively attending to character parts to form appropriate part-based representations in the RH, which may in turn impair orthographic awareness, i.e., knowledge of first-order relations among components. Thus, secondary school students with dyslexia may still encounter difficulties in reading and writing due to persistent deficits in their literacy-related cognitive abilities, and they may require further support to enhance attention to Chinese character components.

Acknowledgements This research was supported by internal funding from EDUHK (project code: RG105/2016-2017R, DRG04244, RG64/2017-2018R, \& PPRCG04297 to R.V. Tso). We are also grateful to the Research Grant Council of Hong Kong (project code: 17402814 to J.H. Hsiao).

Data availability The data that support the findings of this study are available from the corresponding author Dr. Ricky Tso upon reasonable request. Please contact Dr. Ricky Tso through rvytso@eduhk.hk. None of the experiments was preregistered.

\section{References}

Avidan G, Tanzer M, Behrmann M. (2011). Impaired holistic processing in congenital prosopagnosia. Neuropsychologia. 49, 2541-2552.

Behrmann, M., \& Plaut, D. C. (2012). Bilateral hemispheric processing of words and faces: evidence from word impairments in prosopagnosia and face impairments in pure alexia. Cerebral Cortex, bhs390.

Behrmann, M., \& Plaut, D. C. (2013). Distributed circuits, not circumscribed centers, mediate visual recognition. Trends in Cognitive Sciences, 17(5), 210-219.

Bilker, W. B., Hansen, J. A., Brensinger, C. M., Richard, J., Gur, R. E., \& Gur, R. C. (2012). Development of Abbreviated Nine-Item Forms of the Raven's Standard Progressive Matrices Test. Assessment, 19(3), 354-369.

Bosse, M.-L., Tainturier, M.-J., \& Valdois, S. (2007). Developmental dyslexia: The visual attention span hypothesis. Cognition, 140, $198-230$.

Brady, N., Campbell, M., \& Flaherty, M. (2005). Perceptual asymmetries are preserved in memory for highly familiar faces of self and friend. Brain and Cognition, 58, 334-342.
Bukach, C. M., Gauthier, I., \& Tarr, J. M. (2006). Beyond faces and modularity: The power of an expertise framework. Trends in Cognitive Sciences, 10, 156-166.

Chan, C. Y. H., Chan, A. B., Lee, T. M. C., \& Hsiao, J. H. (2018). Eye movement patterns in face recognition are associated with cognitive decline in older adults. Psychonomic Bulletin \& Review, 25(6), 2200-2207.

Chan, D. W., Ho, C. S. H., Tsang, S. M. Lee, S. H., \& Chung K. K. H. (2006). Exploring the reading-writing connection in Chinese children with dyslexia in Hong Kong. Reading and Writing, 19(6), 541561.

Chuk, T., Crookes, K., Hayward, W. G., Chan, A. B., \& Hsiao, J. H. (2017). Hidden Markov model analysis reveals the advantage of analytic eye movement patterns in face recognition across cultures. Cognition, 169, 120-117.

Chung, H. K. S., Leung, J. C. Y., Wong, V. M. Y., \& Hsiao, J. H. (2018a). When is the right hemisphere holistic and when is it not? The case of Chinese character recognition. Cognition, 178, 50-56.

Chung, K. K., \& Ho, C. S. (2010). Dyslexia in Chinese language: An overview of research and practice. Australian Journal of Learning Difficulties, 15(2), 213-224.

Chung, K.K., Lo, J.C., \& McBride, C. (2018b). Cognitive-linguistic profiles of Chinese typical-functioning adolescent dyslexics and highfunctioning dyslexics. Annals of dyslexia, 68, 229-250.

Chung, K. K. H., Ho, C. S.-H., Chan, D. W., Tsang, S.-M., \& Lee, S.-H. (2011). Cognitive skills and literacy performance of Chinese adolescents with and without dyslexia. Reading and Writing, 24(7), 835-859.

Dehaene, S., Cohen, L, Morais, J., \& Kolinsky, R. (2015). Illiterate to literate: Behavioural and cerebral changes induced by reading acquisition. Nature Reviews Neuroscience, 16, 234 - 244.

Facoetti, A., Turatto, M., Lorusso, M., \& Mascetti, G. (2001). Orienting of visual attention in dyslexia: Evidence for asymmetric hemispheric control of attention. Experimental Brain Research, 138, 46 - 53.

Franceschini, S., Bertoni, S., Gianesini, T., Gori, S., \& Facoetti, A. (2017). A different vision of dyslexia: Local precedence on global perception. Scientific Reports, 7(1), 1-10.

Gabay, Y., Dundas, E., Plaut, D., \& Behrmann, M. (2017). Atypical perceptual processing of faces in developmental dyslexia. Brain \& Language, 173, 41-51.

Gabay, Y., Gabay, S., Henik, A., Schiff, R. \& Behrmann, M. (2015). Word and line bisection in typical and impaired readers and a cross-language comparison. Brain and Language, 150, 143-152.

Gabay, Y., Gabay, S., Schiff, R., Ashkenazi, S., \& Henik A. (2013). Visuospatial attention deficits in developmental dyslexia: Evidence from visual and mental number line bisection tasks. Archives of Clinical Neuropsychology, 28, 829-836.

Gauthier, I. \& Bukach, C. (2007). Should we reject the expertise hypothesis? Cognition, 103(2), 322-330.

Gauthier, I., \& Tarr, M. J. (2002). Unraveling mechanisms for expert object recognition: Bridging brain activity and behavior. Journal of Experimental Psychology: Human Perception and Performance, 28(2), 431-446.

Harris, A., \& Aguirre, G. K. (2010). Neural tuning for face wholes and parts in human 43 fusiform gyrus revealed by FMRI adaptation. Journal of Neurophysiology, 104, 44336.

Ho, C. S.-H., Chan, D. W.-O., Chung, K., Tsang, S.-M., Lee, S.-H., \& Fong, C. Y.-C. (2016). The Hong Kong Test of Specific Learning Difficulties in Reading and Writing for Primary School StudentsThird Edition [HKT-P(III)]. Hong Kong: Hong Kong Specific Learning Difficulties Research Team.

Hole, G. J. (1994). Configurational factors in the perception of unfamiliar faces. Perception, 23(1), 65-74.

Hsiao, J. H., \& Cottrell, G. W. (2009). Not all visual expertise is holistic, but it may be leftist: The case of Chinese character recognition. Psychological Science, 20, 455-463. 
Hsiao, J. H., \& Galmar, B. (2016). Holistic processing as measured in the composite task does not always go with right hemisphere processing in face perception. Neurocomputing, 182, 165-177.

Hsiao, J. H., \& Shillcock, R. (2006). Analysis of a Chinese phonetic compound database: Implications for orthographic processing. Journal of Psycholinguistic Research, 35, 405-426

Hsiao, J. H., Shillcock, R., \& Lee, C. (2007). Neural correlates of foveal splitting in reading: evidence from an ERP study of Chinese character recognition. Neuropsychologia, 45, 1280-1292.

Ivry, R., \& Robertson, L. C. (1998). The Two Sides of Perception. Cambridge: MIT Press.

Lachmann T. (2018). Reading and Dyslexia: The Functional Coordination Framework. In: Lachmann T., Weis T. (eds) Reading and Dyslexia. Literacy Studies (Perspectives from Cognitive Neurosciences, Linguistics, Psychology and Education), Vol 16. Springer, Cham

Liu, S., Pan, Z., \& Xu, Z. (2018). The association between reading abilities and visual-spatial attention in Hong Kong Chinese children. Dyslexia, 24(3), 263-275.

Liu, T., Chuk, T., Yeh, S. L., \& Hsiao, J. H. (2016). Transfer of perceptual expertise: The case of simplified and traditional Chinese character recognition. Cognitive Science, 40(8), 1941-1968

Malaspina M, Albonico A, \& Daini R. (2016). Right perceptual bias and self-face recognition in individuals with congenital prosopagnosia. Laterality, 118-42.

Maurer, D., Le Grand, R., \& Mondloch, C. J. (2002). The many faces of configural processing. Trends in Cognitive Sciences, 6, 255-260.

Navon, D. (1977). Forest before trees - Precedence of global features in visual-perception. Cognitive Psychology, 9, 353-383.

Richler, J. J., Tanaka, J. W., Brown, D. D., \& Gauthier, I. (2008). Why does selective attention to parts fail in face processing? Journal of Experimental Psychology: Learning, Memory, and Cognition, 34(6), 1356-1368.

Richler, J. J., Wong, Y. K., \& Gauthier, I. (2011). Perceptual expertise as a shift from strategic interference to automatic holistic processing. Current Directions in Psychological Science, 20, 129-134.
Robbins, R., \& McKone, E. (2007). No face-like processing for objectsof-expertise in three behavioural tasks. Cognition, 103(1), 34-79.

Sandford, A., \& Burton, A. M. (2014). Tolerance for distorted faces: Challenges to a configural processing account of familiar face recognition. Cognition, 132(3), 262-268.

Schmitt, A., Lachmann, A., \& van Leeuwen, C. (2019). Lost in the forest? Global to local interference depends on children's reading skills. Acta Psychologica, 193, 11-17.

Sigurdardottir, H. M., Arnardottir, A., Halldorsdottir, E. T., Omarsdottir, H. R., \& Valgeirsdottir, A. S. (2019). Faces and words are both associated and dissociated: Evidence from visual problems in dyslexia. Retrieved November 23, 2019 from https://doi.org/10. 31234/osf.io/n2kp6

Siok, W. T., Perfetti, C. R., Jin, Z., \& Tan, L. H. (2004). Biological abnormality of impaired reading is constrained by culture. Nature, $431,71-76$.

Stein, J., \& Walsh, V. (1997). To see but not to read; the magnocellular theory of dyslexia. Trends in Neurosciences, 20, $147-152$.

Tan, L. H., Spinks, J. A., Eden, G. F., Perfetti, C. A., \& Siok, W. T. (2005). Reading depends on writing, in Chinese. Proceedings of the National Academy of Sciences, USA, 102, 8781-8785.

Tse, S. K. (2011). Effective Learning of Chinese Characters through Discerning and Combining Character Components. Han Character and Han Writing Education, 26, 45-62.

Tso, R. V., Au, T. K., \& Hsiao, J. H. (2014). Perceptual Expertise: Can Sensorimotor Experience Change Holistic Processing and Left-Side Bias? Psychological Science, 25, 1757-1767.

Wolf, M., \& Bowers, P. (1999). The "Double-Deficit Hypothesis" for the developmental dyslexias. Journal of Educational Psychology, 91, 124.

Publisher's note Springer Nature remains neutral with regard to jurisdictional claims in published maps and institutional affiliations. 